\title{
El capital social como recurso de innovación para la gestión regional en grandes áreas protegidas. La Reserva de la Biósfera Großes Walsertal'
}

\author{
Falk F. Borsdorf ${ }^{2}$
}

\begin{abstract}
RESUMEN
La gestión regional en contextos como las áreas protegidas, demandan una continua vinculación de los actores locales y la ciudadanía. Estos grupos no son solo consumidores, sino que también coconstructores de su paisaje cultural. Para asegurar su ayuda constante, la gestión regional tiene que considerar instrumentos que garanticen una cooperación a largo plazo. En este punto es donde el capital social entra en juego y contribuye a crear un compromiso a largo plazo. La conciencia y el énfasis en el capital social pueden tener un gran impacto en las redes regionales de un área de montaña protegida. Aunque no siempre existió la intención deliberada de usar su conocimiento de capital social, el caso de la Reserva de la Biósfera Großes Walsertal, se sitúa como un buen ejemplo de compromiso público a largo plazo, el cual es transformado en significativos grados de innovación y éxito económico.
\end{abstract}

Palabras clave: Capital social, áreas protegidas, desarrollo regional innovativo.

\begin{abstract}
Regional management of regional contexts such as Protected Areas demands the continuous involvement of local stakeholders and citizens. These groups are not only consumers, but also co-constructors of their cultural landscapes. To ensure their persistent support, however, regional management has to take into account instruments likely to guarantee this long-term public support. This is where social capital comes into play and contributes to voluntary engagement on a long-term basis. Though not always having intended to use its knowledge on social capital, the case of the Großes Walsertal Biosphere Reserve appears to have done a good job in bringing about this long-term public support while turning it into significant degrees of innovation and economic success.
\end{abstract}

Key words: Social Capital, Protected Areas, Innovative Regional Development.

1 El autor agradece a Carla Marchant por su colaboración en la traducción del texto y a Theresia Dummer por su asistencia en la elaboración de las figuras. Artículo recibido el 3 de marzo de 2012, aceptado el
3 de noviembre de 2012 y corregido el 12 de enero de 2013

2 alpS Centre for Climate Change Adaptation Technologies (Austria). E-mail: fborsdorf@yahoo.de 
Los procesos de desarrollo regional en áreas protegidas enfrentan un desafío fundamental: estos deben ser encabezados por las personas que viven en ellas. Los habitantes de una determinada región se encuentran en el supuesto de actuar, no solo como consumidores, sino que también como productores del paisaje cultural y por tanto son exhortados a apoyar y ser parte del proceso de desarrollo regional. Mientras el concepto tradicional de gestión regional puede ser relativamente fácil de implementar a través de organizaciones administrativas regionales, con la participación y apoyo de la población pueden convertirse rápidamente en una barrera infranqueable. Asimismo, si los administradores de las áreas protegidas no toman en cuenta el potencial de la región para constituir redes de apoyo, la capacidad de trabajar en conjunto y la existencia de un sistema de valores, esta situación se complica aún más. Lo anterior tiene como resultado, que el desarrollo regional no conducirá a nada y la gestión se realizará de espaldas a la región, sin que esta participe y dirija el proceso. Por esta razón, es particularmente importante para las áreas protegidas el impulso del capital social.

El concepto de capital social reúne no solo aspectos estructurales, como por ejemplo la relación entre actores, sino que abarca también el intercambio entre personas individuales y grupos y los resultados que se producen en dicho intercambio. Así, es posible suponer que un grado significativo del capital social de una comunidad, ya sea de un valle, un pueblo o un proyecto de desarrollo regional, puede ser considerado como un recurso que puede contribuir a la superación de determinados desafíos. Asimismo, un conjunto de normas y valores contribuyen al desarrollo del capital social, fortaleciéndolo y consolidándolo, ejemplos de ello son la confianza y la reciprocidad entre los participantes del proceso (de desarrollo regional u otras situaciones similares) (Ostrom \& Ahn, 2009). Siguiendo a Putnam (2000: 90), el capital social "hace a las personas más inteligentes, saludables, seguras, ricas y más capaces para gobernar una democracia más justa y estable". A través de la fusión de elementos como el establecimiento de redes y la adopción de normas y valores orientados a la comunidad, se refuerzan las relaciones sociales. La participación en redes, agrupaciones o asociacio- nes comunitarias, como también el contacto en el lugar de trabajo, con amigos o parientes actúan como agentes que fortalecen el espíritu cívico de una sociedad. El enriquecimiento de las relaciones sociales con valores como la orientación democrática, la solidaridad, la tolerancia y la reciprocidad, puede producir una profundización en el entendimiento del conjunto de normas y valores del capital social (Jungbauer-Gans, 2006). Por lo general, el capital social se presenta como un recurso público que combina redes sociales, normas, valores y confianzas. En el marco de procesos de desarrollo regional, una mayor conciencia y una consecuente promoción del capital social puede contribuir a una planificación más adecuada y específica de la gestión regional, con una mayor participación y visión de largo plazo.

En el marco de proyectos de desarrollo regional, es posible afirmar que aquellos individuos que poseen comportamientos orientados a la comunidad, están también más dispuestos a contribuir activamente en estos procesos, con la expectativa de que sus esfuerzos sean reconocidos por los demás, en lugar de ser explotados. Cuando la confianza es recíproca, es posible reducir costos en la gestión regional, por ejemplo en la etapa de preparación de contratos, contratación de abogados, etc. (Jungbauer-Gans, 2006: 27). Por esta razón, la confianza es considerada como

"el vínculo fundamental entre el capital social y la acción colectiva. La confianza se incrementa cuando las personas son honestas, trabajan en equipo y están vinculadas con instituciones que son dignas de confianza [...] Densas redes horizontales, conocidas como capital social cohesivo, con la capacidad de transmitir eficientemente información a través de los miembros de la red y también crear incentivos para mantener un clima de confianza incluso para aquellos que tienen motivaciones egoístas" (Ostrom \& Ahn, 2009: 22-24).

Por esta razón, el capital social puede hacer una contribución esencial para el éxito de procesos de desarrollo regional sustentable.

Una gestión regional, que en el marco de proyectos de desarrollo regional considera el 
capital social, será seguramente apoyada por un amplio sector de la población. Las innovaciones que pueden surgir en estos procesos, contribuyen a profundizar la confianza y a estrechar las redes sociales (Borsdorf, 2011). La población regional tendrá rápidamente la impresión que tiene un rol en los procesos de largo plazo y que pueden influenciar en ellos y por consiguiente construir su espacio vital (Trattnigg, 2008a, 2008b). Por esta razón, la estimulación de un enfoque de comunidad, es decir, el capital social, es una tarea fundamental para la gestión regional de áreas protegidas (Borsdorf, 2010). Como es sabido, numerosos estudios señalan la importancia en la biografía de las personas, del desarrollo de un sentimiento de pertenencia a una comunidad "en el hogar, en la escuela, en el trabajo, en asociaciones de voluntariado o en instituciones religiosas, las personas adquieren recursos, reciben solicitudes para actividades y desarrollan orientaciones políticas que promueven la participación" (Burns et al., 2001: 35). Estas orientaciones influencian y tienen también un efecto en el marco de procesos de desarrollo regional sustentable.

Este artículo analiza el ejemplo de la Reserva de la Biósfera Großes Walsertal, Iocalizada en un valle de la región de Walgau del Estado Federal austriaco de Vorarlberg, en la cual es posible identificar un capital social que puede ser aprovechado para un desarrollo sostenible con diferentes grados de innovación y éxito económico.

\section{Aspectos de la gestión regional exitosa de grandes áreas protegidas}

El concepto de desarrollo regional puede perseguir distintas metas. En este contexto, las posibilidades abarcan desde el fortalecimiento de la competitividad regional y/o de la situación económica, a la concentración de proyectos individuales para la elaboración de una estrategia regional, o la motivación de los actores regionales para promover la acción colectiva (Toben, 2007). En este contexto se reconocen tres fases: análisis, concepto y la fase de acción. En la fase de análisis se recolectan los antecedentes principales de la región (economía, población, desarrollo del mercado de trabajo) y se realiza un análisis
FODA, para facilitar al grupo de trabajo la definición de los objetivos y metas en los cuales debe enfocarse el proceso de desarrollo. Al concluir esta fase se elabora un concepto, esto es una imagen creada conjuntamente, sobre la cual se orienta el desarrollo regional futuro. A continuación, se inicia la fase de acción, donde la teoría debe llevarse a la práctica a través de proyectos concretos de desarrollo regional. Para asegurar estos procesos, la región necesita además de una forma jurídica, un gestor o líder permanente que se haga cargo de estas tareas. Este apoya la ejecución de los proyectos, participa en la búsqueda de financiamiento y establece una red de contactos con los principales tomadores de decisiones de la región. Asimismo, participa en las relaciones públicas del proceso, vela por la aplicabilidad del concepto de desarrollo, mantiene activa y coordina la gestión regional, por ejemplo a través del desarrollo de mesas de trabajo y seminarios de temas concretos de interés (Toben, 2007). Para la realización de estas tareas, normalmente es necesario contar con uno o más trabajadores.

Como en muchos otros casos, es válido también para la gestión regional de áreas protegidas, el desarrollo de un enfoque metodológico, que se utiliza normalmente como se observa en el Cuadro $N^{\circ} 1$. El control, la organización, estructuración y documentación de los procesos de desarrollo regional son parte de las tareas fundamentales de la gestión regional (Grabski-Kieron, 2002). Asimismo, se cuenta el análisis regional (imagen objetivo y lineamientos de desarrollo), planificación de las estrategias de comunicación (fomento del medio ambiente regional, asesoramiento en la fase de planificación y puesta en marcha) y el control y seguimiento del proyecto (determinación del punto cúlmine del desarrollo regional, uso eficaz de los recursos, apoyo a la implementación y coordinación). El punto más importante de estas complejas tareas es sin duda el marketing regional, el cual es definido por Manschwetus (1995:39) como un concepto de control orientado al mercado, el cual se utiliza para el desarrollo de una región. La idea central del marketing regional es la construcción de una relación entre la región y los socios del mercado. En consecuencia, la mayoría de los recursos utilizados son medios de comunicación (folletos, difusión en Internet, campañas 
Cuadro $\mathrm{N}^{\circ} 1$

Enfoque metodológico del concepto de gestión regional

\begin{tabular}{|l|l|}
\hline \multicolumn{1}{|c|}{ Tareas } & \multicolumn{1}{c|}{ Efectos } \\
\hline Gestión regional & $\begin{array}{l}\text { control, organización, estructuración, documentación de un proceso de } \\
\text { desarrollo regional }\end{array}$ \\
\hline Análisis regional & Objetivos y líneas de evolución \\
\hline $\begin{array}{l}\text { Comunicación de } \\
\text { planeación }\end{array}$ & $\begin{array}{l}\text { Promoción de medios sociales regionales, asesorías en procesos de } \\
\text { planeación y la realización de los planes }\end{array}$ \\
\hline Marketing regional & $\begin{array}{l}\text { Promoción de identidad regional, posicionamiento de la región en la } \\
\text { competición de regiones }\end{array}$ \\
\hline $\begin{array}{l}\text { Control de } \\
\text { proyectos }\end{array}$ & $\begin{array}{l}\text { Punto de cristalización de desarrollo regional, utilización de fondos } \\
\text { efecto, promoción de realización, coordinación }\end{array}$ \\
\hline
\end{tabular}

Fuente: Modificado a partir de Grabski-Kieron, 2002.

publicitarias), con el fin de posicionar a la región con sus ventajas comparativas ante otras regiones vecinas. A menudo se utilizan también productos regionales, que no solo entregan una imagen sobre ella, sino que establecen una marca y aseguran la identidad regional (Toben, 2007; Köstlin, 2010). Debido a que los habitantes y usuarios de una región, en este caso, de un área protegida, no solo deben ser considerados consumidores, sino que también productores de su paisaje cultural, los encargados del manejo regional necesitan un profundo conocimiento del capital social de la región. Como se argumentará más adelante, el factor capital social, debe ser considerado como un elemento fundamental más del concepto tradicional de gestión regional.

\section{El capital social como un nuevo pilar}

La práctica de la gestión regional de áreas protegidas ha demostrado que a largo plazo, las formas regionales de cooperación y participación en el marco de procesos de desarrollo regional sustentable, sin la presencia de ciudadanos comprometidos, no son posible de sostener en el tiempo (Stoll-Kleemann \&
Welp, 2008; Lahner, 2009; Elbe, 2011; Horlings, 2010). Experiencias similares se han repetido en numerosos procesos locales de implementación de la Agenda 21, señalándose que estos procesos de participación a largo plazo requieren la contribución activa de los ciudadanos locales (Geißel \& Kern, 2000). Cuando se parte de un concepto de sustentabilidad integrativa, es necesario que el modelo de participación ciudadana impulse el desarrollo regional, sin que el capital social disponible y con ello, una parte de población activa, sea desaprovechada (Trattnigg, 2008a, 2008b; Geißel \& Kern, 2000). En numerosas ocasiones, la práctica ha demostrado que el objetivo de poseer una amplia participación de ciudadanos de una comunidad perdurable a largo plazo, no es posible sin que estos individuos estén comprometidos y sin la existencia de capital social disponible (Lahner, 2009; Stoll-Kleemann \& Welp, 2008). En este punto es posible recalcar, que una verdadera cultura de la sustentabilidad no es posible de desarrollar donde los elementos centrales del capital social, como la construcción de estrechas redes de colaboración, existencia de capital social vinculante, o confianza, no existen o estas son muy débiles (Trattnigg, 2008: 88). El ideal de un capital social sustentable, el cual apoya y encabeza procesos de desa- 
rrollo regional a largo plazo, es observable en un modelo de autoorganización. Sin la coacción institucional para la cooperación, estos procesos necesitan de normas alternativas de integración, las cuales pueden producir capital social y apoyarlo; de otro modo, una acción colectiva de largo plazo será difícil de implementar (Lahner, 2009: 35). Lo anterior deja en evidencia que en el contexto regional la disponibilidad de capital social posee una gran relevancia para el éxito a largo plazo de los procesos de desarrollo regional.

El medio ambiente y el paisaje de una región, vistos como una base común para un futuro sustentable, permite a la comunidad compartir un punto común y fortalecer el sentimiento de comunidad y por tanto también el capital social (Fürst et al., 2006). Las reservas de la biósfera tendrían en este caso, el potencial de ser importantes excepciones a la "tragedia de los comunes", propuesta por Garrett Hardin (1968). Con capital social, unidad, redes de colaboración, sentimiento de comunidad y confianza, la tarea de conservar y desarrollar sosteniblemente el paisaje cultural se constituye en el núcleo central de lo que se denomina desarrollo regional sustentable. Esto supone que en los organismos encargados de desarrollar la gestión regional, tienen conocimiento del alcance y las formas de capital social existentes en la región, el cual puede ser utilizado para la planificación de procesos de desarrollo regional.

Entendida como la tarea central de la gestión regional, la promoción del capital social en un área protegida, puede ser un impulso positivo a las innovaciones, las cuales pueden conducir también a una bonanza económica. Así lo demuestran numerosos estudios sobre capital social e implementación de medidas de innovación regional, estas últimas pueden ser reforzadas a través de distintas características del capital social (Hauser et al., 2007). La región ofrece aquí un marco de referencia confiable, que normalmente proporciona estructuras económicas homogéneas (Renn \& Kastenholz, 1996: 97). La cooperación entre productores y proveedores, como también entre empresas regionales y el gobierno local, mejora sustantivamente cuando en el ambiente de negocios prima un clima de confianza (Blättel-Mink, 2006). Las redes sociales concebidas para actuar a largo plazo, pueden también en otros aspectos contribuir a desarrollar innovaciones para el desarrollo regional. Especialmente en áreas de débil desarrollo, la promoción del capital social puede tener un rol decisivo en la mejora de los servicios públicos (Favry et al., 2006), como también puede dar lugar a la creación de sistemas de innovación regional, las cuales pueden transformar las desventajas en oportunidades.

Es ampliamente reconocido, que la construcción de "redes de colaboración útiles" (Holzer, 2006: 14), es imprescindible para el desarrollo e implementación de un área protegida y también es fundamental para su funcionamiento futuro. Como se demostrará en las líneas siguientes, la percepción de una crisis en Großen Walsertal, pudo conducir exitosamente, a través del establecimiento de una Reserva de la Biósfera, a la creación de una región modelo para el desarrollo sustentable (Reutz-Hornsteiner, 2009; Dax et al., 2009). Resulta importante mencionar que, el conocimiento del alcance y las formas del capital social en el valle, durante la fase de planificación, no se encontraba todavía debidamente identificado. Sin embargo, es necesario recalcar, que solo un fuerte "componente social" con lazos de amistad estrechos y un marcado sentimiento de comunidad en la población, además de confianza y apoyo mutuo, puede conducir a procesos exitosos de desarrollo en reservas de la biósfera.

\section{El capital social concreto: el ejemplo de la Reserva de la Biósfera Großes Walsertal}

La Reserva de la Biósfera Großes Walsertal es un interesante ejemplo de cómo el capital social existente en una región y el trabajo en comunidad, juegan un rol fundamental para la gestión de áreas protegidas. Esta reserva se ubica en un entorno de marcadas características de ruralidad, al oeste de Austria y desde el año 2000, gracias al esfuerzo conjunto de los habitantes del valle, posee el título de Reserva de la Biósfera, el cual es otorgado por la Organización de las Naciones Unidas para la Educación, la Ciencia y la Cultura (UNESCO). Lo anterior ocurrió en el contexto de una profunda reflexión, sobre cómo era posible sacar al valle de la crisis en 
la que se encontraba y sobre todo buscar soluciones para problemáticas esenciales, como la infraestructura deficiente, la falta de trabajo en la región, el gran número de población migrante diaria, el difícil acceso al transporte público y una población de edad mayoritariamente avanzada, etc. (Fritsche \& Studer, 2009). Como objetivo central se estableció, convertir al valle Großes Walsertal en una región modelo para el desarrollo sustentable e impulsarla a través de una nueva imagen. Por ello el modelo de reserva de la biósfera ofrecido por la UNESCO pareció ser la solución más adecuada para alcanzar esta meta. La admisión del valle significó el ingreso a la red mundial de reservas de la biósfera (ReutzHornsteiner, 2009: 18). El proceso de aprobación fue rápidamente obtenido, debido a que los encargados de iniciar el proceso de planificación convocaron de manera efectiva la participación pública, sin olvidar a ninguno de los actores relevantes. La recién establecida organización de planificación regional REGIO, compuesta por los alcaldes de seis comunas pertenecientes al valle, tuvieron un rol de plataforma central. Como en muchos otros casos de regionalización de la cultura, es necesaria la existencia de un líder que se encargue de comandar estos procesos de cambio (Köstlin, 1980). En el caso del valle Großes Walsertal, este liderazgo recayó en el miembro del parlamento, Josef Türtscher, quien fue el encargado de crear una visión conjunta y traducir esta en acciones. Gracias a todos estos esfuerzos, en el año 2000 el valle fue incorporado a la red mundial de la UNESCO de reservas de la biósfera (Figura $\left.\mathrm{N}^{\circ} 1\right)$.

Es interesante recalcar que para esta resolución rápida y exitosa, fue fundamental el hecho de que los actores participantes tuvieran un buen conocimiento de su región y de la cohesión social existente en ella. Hasta el día de hoy es posible apreciar en Großes Walsertal un profundo sentimiento de comunidad, pertenencia e identidad, el cual para muchos proyectos de desarrollo regional emerge como un factor que los influencia de manera positiva (Jungmeier et al., 2010). Este

Figura $\mathrm{N}^{0} 1$

Mapa de localización: Reserva de la Biósfera Großes Walsertal

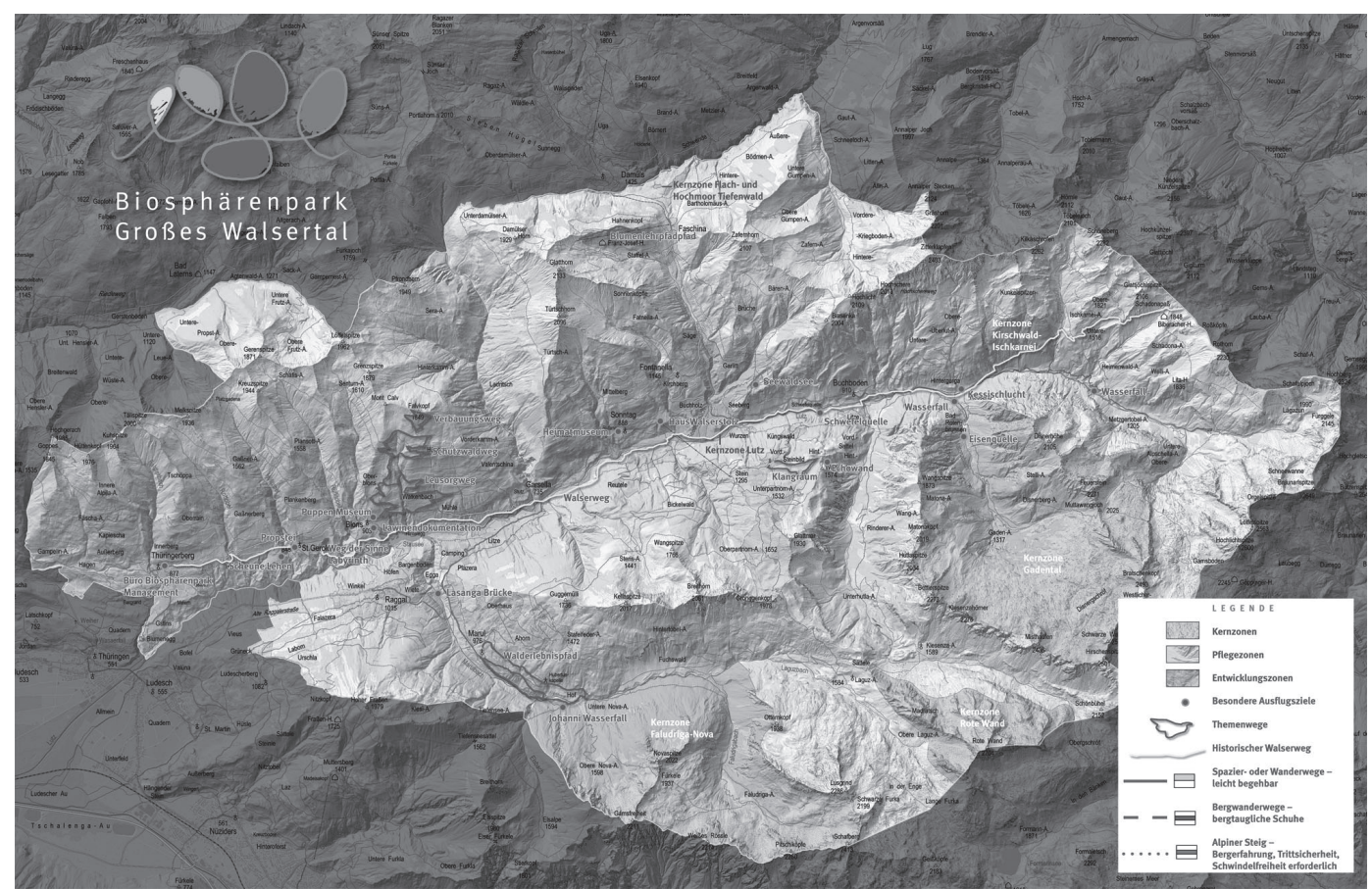

Fuente: Elaboración propia. 
hecho sugiere, también, que existen muchas personas individuales comprometidas y asimismo un número importante de iniciativas regionales en el valle, para los cuales su vinculación puede ser positiva, por tanto este potencial debe ser aprovechado para la implementación de proyectos de desarrollo regional.

De manera general, las áreas protegidas parecen ser un lugar muy fructífero para el desarrollo del capital social. En este caso particular, Großes Walsertal se encuentra bajo la administración del estado federado de Voralberg, el cual cuenta con una oficina encargada de estrategias de futuro, la cual hace ya varios años se ocupa de temas relacionados al fomento del capital social y a impulsar proyectos de desarrollo regional. En este contexto, la oficina desarrolla estudios de capital social, tanto a nivel de estado federado, como a nivel local. En el nivel federal la oficina busca mejorar las redes de participación ciudadana, así también promueve una mejor coordinación, comunicación y trabajo conjunto entre políticos, municipios, empresas, asociaciones y escuelas (Hellrigl, 2006: 93). En resumen, la gestión de la reserva de la biósfera fue capaz de aprovechar las condiciones favorables y el ambiente positivo de la región, como también del fuerte sentimiento de comunidad existente, lo cual tuvo consecuencias positivas para la implementación de este proyecto.

El tercer y más reciente estudio sobre capital social desarrollado por la oficina de estrategias de futuro tuvo por objetivo establecer las bases para un monitoreo a largo plazo del capital social en Voralberg. Basándose en una muestra representativa, el estudio desarrollado en el año 2010, concluyó que el capital social y la participación ciudadana en Voralberg son excepcionalmente fuertes, en este sentido, cuatro de seis de las dimensiones de la participación cívica y ocho de las nueve dimensiones del capital social obtuvieron altas puntuaciones en el estudio cuantitativo. La educación y la situación laboral, que normalmente son factores decisivos para el capital social, tuvieron un rol secundario (Fredersdorf et al., 2010: 4 ff.). Lo anterior demuestra una clara tendencia a la existencia a nivel de estado federado, de un capital social y un sentimiento de comunidad fuerte, el cual es posible también advertir en el valle Großen Walsertal.

Esta idea se manifiesta en el contexto de las redes densas (también Ilamado capital social cohesivo), donde se afirma que es posible encontrar soluciones para los problemas intrarregionales de manera conjunta (Fritsche \& Studer, 2009: 15). El mejor ejemplo de ello fue la idea elaborada conjuntamente, de transformar al valle en una región modelo para el desarrollo sustentable, en la cual tanto los numerosos actores sociales, como las personas individuales comprometidas, pudieran participar (Reutz-Hornsteiner, 2009). En este contexto resulta destacable el número de organizaciones existentes en la región, contabilizándose un total de 111 (Fritsche \& Studer, 2009: 83 ff.). En lo relativo a cooperación intermunicipal, la región es un ejemplo; las seis municipalidades construyeron una plataforma de trabajo común, para coordinar las formas públicas y privadas de cooperación y colaboración, las cuales sentarían las bases para el establecimiento de la reserva de la biósfera (Reutz-Hornsteiner, 2006). De esta forma, el intercambio entre los comités y gremios de REGIO (agricultura, educación, escuelas y cultura, turismo, comercio, medio ambiente, servicios sociales y juventud), pudo establecer vínculos entre ciudadanos y políticos comunales, los cuales fortalecieron la economía regional y las diversas organizaciones e iniciativas desarrolladas en el valle, aumentando asimismo la confianza entre ellos. De la misma forma, la fusión de las funciones administrativas ha marchado exitosamente en algunos aspectos, siendo un ejemplo destacable para las reformas administrativas en el Estado austriaco. El uso de un espacio común (como el Bauhof en Raggal) compartido por las seis comunas, no es solo un ahorro de dinero para los gobiernos locales, sino que también favorece la comunicación entre comunidades.

El capital social vinculante no fue un tema relevante en el valle, debido a que en este la estructura social es homogénea (Fritsche \& Studer, 2009). Este hecho es positivo, dado que esta característica puede ser relevante en los procesos de desarrollo regional. En este contexto, una característica importante del capital social en Voralberg es el limitado papel que tiene tanto el nivel de educa- 
ción, como la situación laboral (Fredersdorf et al., 2010). Asimismo, la gran diversidad de ramas de ocupación existentes en el valle y de organizaciones ligadas a ellas, permiten fortalecer la integración y alimentar los lazos entre una gran cantidad de población, fomentando diversas formas de la cooperación y trabajo conjunto.

Como algunos autores han demostrado, la reserva de la biósfera es un modelo que posee un grado de aceptación muy alto: un $82,4 \%$ de los participantes de una encuesta realizada en 2007 (universo de encuestados: 532) expresaron su aprobación a este modelo. Del mismo modo, un alto porcentaje manifestó participar activamente en proyectos de desarrollo regional: un $25,2 \%$ de los entrevistados ha participado alguna vez en alguno de los workshops, asimismo un $40 \%$ manifiesta su disposición a participar, si el tema es de su interés (Figura $\mathrm{N}^{\circ} 2$ ). Lo anterior deja en evidencia una sólida confianza de la población en el nuevo contexto regional en que se encuentran (Rumpolt, 2009: 52). Este resultado toma aún más fuerza, si se compara con el promedio nacional de Austria en materias de participación; de acuerdo a Plasser \& Ulram (2010: 167-168) cerca de un 30\% de los austriacos tiene una participación activa en alguna iniciativa y estarían dispuestos a participar en ideas no convencionales. En este contexto, para la gestión de reservas de la biósfera parece esencial fomentar desde el inicio la integración de la población en la creación, tanto de la imagen objetivo, como de los proyectos de desarrollo regional (Weixlbaumer \& Coy, 2009); con ello es posible fortalecer la confianza, tanto interpersonal, como entre ciudadanos y sus representantes locales. En la fase de creación de la imagen objetivo, 70 personas participaron activamente, lo cual permite comprobar lo anteriormente señalado (Reutz-Hornsteiner, 2009: 21). Igualmente beneficioso resultó ser contar los líderes de opinión claves de la región, lo cual tuvo un efecto positivo para aumentar la confianza de la población del valle (Reutz-Hornsteiner, 2009). Finalmente, y en estrecha relación con el punto anterior, es posible mencionar también como elementos positivos la congruencia tanto en la autoevaluación, como en la evaluación externa realizada al funcionamiento del área protegida, factor que cuenta como un indicador de expectativas realistas y de un alto grado de autoconfianza (Weixlbaumer \& Coy, 2009).

De acuerdo a lo que señalan los resultados obtenidos en una gran variedad de estudios de capital social, existen importantes diferencias de género en esta materia. Mientras que los hombres se organizan en asociaciones comunitarias $y / 0$ clubes, las mujeres lo hacen de manera más informal (Fredersdorf et al., 2010). Los resultados obtenidos en Voralberg coinciden y reflejan muy bien la situación a nivel país, cuando se trata de orientaciones comunes (More-Hollerweger \& Heimgartner, 2009). La misma tendencia es posible observar a nivel regional, en la Reserva de la Biósfera Großen Walsertal, donde existen más hombres que mujeres participando de asociaciones. Para revertir esta situación, se desarrolló un proyecto para fortalecer la participación femenina, el cual consistió en el desarrollo de un huerto de hierbas ornamentales (Alchemilla) (Moser, 2009). De manera general, es posible señalar que en la Reserva de la Biósfera Großen Walsertal existe una división especial del trabajo: mientras los comités de REGIO se encuentran compuestos mayoritariamente por hombres (a excepción del grupo de trabajo de temas sociales), la gestión del área protegida recae mayoritariamente en mujeres. Este tipo de estructura es posible de encontrar en Austria en muchos otros proyectos, procesos e iniciativas (Oedl-Wieser, 2010). Lo anterior da cuenta que el manejo de la reserva de la biósfera y la presencia de modos informales de capital social, pueden ser utilizados como un recurso disponible para la implementación de una serie de proyectos de desarrollo regional.

De la misma forma en que se desarrollaron los campos tradicionales de la gestión regional, también se impulsaron proyectos innovadores en Großen Walsertal, los cuales demostraron ser compatibles. El ejemplo más importante en esta materia, es el desarrollo de un queso de montaña, conocido como el Walserstolz (orgullo del valle de Walser), el cual no solo conllevó un beneficio económico, sino que también entregó al valle un valor agregado especial. Con la creación de una lechería comunitaria en el año 1998, es decir antes de la fundación de la reserva de la biósfera, se buscó consolidar la fuerza regional. 
La producción de este tipo especial de queso, con una impronta regional, fue posible garantizar altos precios de la leche y la promoción de la identidad regional. Asimismo, la imagen del producto es relacionada con características regionales, como tradición, amor y trabajo colectivo. Desde la perspectiva económica, se produjo un aumento de un $10 \%$ en los ingresos de las lecherías, una menor necesidad de inversión para los campesinos, la conservación del paisaje cultural del valle y también se han obtenido por ello diversos premios. Con el apoyo de proyectos de desarrollo regional, impulsados por la reserva de la biósfera, se logró la implementación de proyectos de comercialización e implementación de cadenas de suministro, como por ejemplo a través de una iniciativa desarrollada en conjunto con empresarios gastronómicos y productores, la cual se orientó a posicionar los productos de alta calidad en restaurantes en diferentes destinaciones turísticas, para así brindar a los huéspedes una experiencia culinaria inolvidable. Por otro lado, la producción del queso se desarrolla en tres lecherías en el valle y en diez cabañas en la montaña. Desde el lanzamiento de la marca Walserstolz en el año 1998, las ganancias han aumentado seis veces (Weixlbaumer, 2010: $37 \mathrm{ff}$.). Contrariamente a lo que muestra la experiencia de otros casos de relocalización de la producción de alimentos, la cual a menudo genera mayor valor agregado (Schermer, 2010: 25 ff.), el mantenimiento de la cadena de producción de queso Walserstolz en el valle, condujo a fomentar de manera positiva y sustentable la imagen de este. El desarrollo de un mercado, la comercialización y exportación pudieron en este sentido, generar un efecto positivo en el desarrollo del paisaje cultural y hacer más fuerte el impulso innovativo de los actores locales (Weixlbaumer, 2010: 38). Sin necesariamente pretenderlo, el capital social disponible de una región puede ser utilizado para desarrollar nuevos productos con un alto grado de innovación. La confianza, generosidad y empeño condujeron a un nuevo éxito regional.

\section{Conclusión}

El conocimiento del capital social disponible en una región puede convertirse en un factor clave para el éxito de la gestión regional de un área protegida. Incluso en re- giones donde el capital social es utilizado sin saberlo directamente, puede el uso de estas y otras estrategias conducir a proceso exitosos. Este era el caso de la Reserva de la Biósfera Großen Walsertal, la cual comenzó su transformación en un área protegida a través de procesos enfocados "desde abajo", es decir, a través de la vinculación de los principales tomadores de decisiones y actores locales involucrados y también gracias a la implementación de una serie de innovaciones. Lo anterior demuestra que mientras existen resultados concretos, la gestión regional de la Reserva de la Biósfera Großen Walsertal puede disponer del capital social existente en la región para emprender e implementar nuevos proyectos de desarrollo regional. Como muestra la experiencia en este caso y en general para la gestión regional, es claro que procesos de desarrollo a largo plazo solo pueden construirse con la vinculación de los diferentes tomadores de decisiones y actores locales y también con resiliencia para enfrentar de manera efectiva los desafíos de nuestro tiempo actual. El secreto del éxito de una gestión regional innovadora es el uso constante y la promoción del capital social existe en una región.

\section{Referencias bibliográficas}

BLÄTTEL-MINK, B. Kompendium der Innovationsforschung. Wiesbaden: Springer Fachmedien, 2006.

BORSDORF, F. Regional Management is Social Work! Activating social capital as a key task of regional managers - the case of the Biosphere Reserve Wienerwald. In: BORSDORF, A.; GRABHERR, G.; HEINRICH, K.; SCOTT, B. \& STÖTTER, J. (editors). Challenges for Mountain Regions - Tackling Complexity. Vienna: Österreichischen Akademie der Wissenschaften, 2010, p. 162-169.

BORSDORF, F. Social Capital as a key source for sustainable development in protected mountain areas: experiences from the Großes Walsertal Biosphere Reserve. In: BORSDORF, A.; STÖTTER, J. \& VEULLIET, E. (editors). Managing Alpine Future II: Proceedings of the Innsbruck Conference. Vienna: Österreichischen Akademie der Wissenschaften, 2011, p. 427-439. 
BURNS, N.; SHLOZMAN, K.L. \& VERBA, S. The Private Roots of Public Action. Cambridge: Cambridge University Press, 2001.

DAX, TH.; FAVRY, E.; FIDLSCHUSTER, L.; OEDL-WIESER, TH. \& PFEFFERKORN, W. Neue Handlungsmöglichkeiten für periphere ländliche Räume. Stärkung der sozialen Vielfalt, Ausbau der interkommunalen Zusammenarbeit, Gestaltung der Landschaftsvielfalt. Wien: ÖROK, 2009.

ELBE, J. Die Wirksamkeit von Sozialkapital in der Regionalentwicklung. Erprobung eines anwendungsorientierten Analysekonzepts am Beispiel des Modellvorhabens "BioenergieRegionen". Aachen, 2011.

FAVRY, E.; HIESS, H.; MUSOVIC, Z.; SMRZKA, B. \& PFEFFERKORN, W. Aufrechterhaltung der Funktionsfähigkeit Ländlicher Räume. Dienstleistungen der Daseinsvorsorge und Regional Governance: Veränderungen, Herausforderungen, Handlungsbedarf. Wien, 2006.

FREDERSDORF, F.; ROUX, P. \& LORÜNSER, D. Endbericht zur Studie Bürgerschaftliches Engagement und Sozialkapital in Vorarlberg 2010 - Soziales Monitoring für Sozialkapital und Engagement. Dornbirn: Band 1, Hauptergebnisse, 2010.

FRITSCHE, A. \& STUDER, L. Lebenswelt Großes Walsertal. Regensburg: Institut für sozialwissenschaftliche Regionalforschung, 2009.

FÜRST, D.; LAHNER, M. \& POLLERMANN, K. Entstehung und Funktionsweise von Regional Governance bei dem Gemeinschaftsgut Natur und Landschaft. Analysen von Place-Making und Governance-Prozessen in Biosphärenreservaten in Deutschland und Großbritannien. Hannover: Schriftenreihe der Fachgruppe Landschaft, Beiträge zur räumlichen Planung, Band 82, 2006.

GEIßEL, B. \& KERN, C. Soziales Kapital und Lokale Agenda 21. In: HEINELT, H. \& MÜHLICH, E. (Hg.). Lokale Agenda 21-Prozesse: Erklärungsmuster, Konzepte und Ergebnisse. Opladen, 2000, p. 257-275.
GRABSKI-KIERON, U. Die Bedeutung des Regionalmanagements für die nachhaltige Entwicklung ländlicher Räume. Landentwicklung Aktuell, Heft 2001/2002, p. 11-13.

HARDIN, G. The Tragedy of the Commons. Science, 1968, $\mathrm{N}^{\circ} 162$, p. 1.2431.248 .

HAUSER, CH.; TAPPEINER, G. \& WALDE, J. The Learning Region: Impacts of Social Capital and Weak Ties on Innovation. Regional Studies, 2007, Vol. 41, № 1, p. 75-88.

HELLRIGL, M. Zukunftsfrage Sozialkapital. In: GEHMACHER, E.; KROISMAYR, S.; NEUMÜLLER, J. \& SCHUSTER, M. (Hg.): Sozialkapital. Neue Zugänge zu den gesellschaftlichen Kräften. Wien: Mandelbaum-Verlag, 2006, p. 93-105.

HOLZER, B. Netzwerke. Bielefeld: transcript, 2006.

HORLINGS, I. How to generate sustainable European Rural Regions: The role of Social Capital, Leadership and Policy Arrangements. Regions, 2010, №280, p. 8-12.

JUNGBAUER-GANS, M. Einleitende Betrachtungen zum Begriff Sozialkapital. In: GEHMACHER, E.; KROISMAYR, S.; NEUMÜLLER, J. \& SCHUSTER, J. (Hg.). Sozialkapital. Neue Zugänge zu den gesellschaftlichen Kräften. Wien: Mandelbaum-Verlag, 2006, p. 17-43.

JUNGMEIER, M.; PAUL-HORN, I.; ZOLLNER, D.; BORSDORF, F.; LANGE, S.; REUTZHORNSTEINER, B.; GRASENICK, K.; MOSER, R. \& DIRY, C. Part_b: Partizipationsprozesse in Biosphärenparks - Interventionstheorie, Strategieanalyse und Prozessethik am Beispiel vom Biosphärenpark Wienerwald, Großes Walsertal und Nationalpark Nockberge. Band 1: Zentrale Ergebnisse. Klagenfurt: Österreichische Akademie der Wissenschaften, 2010.

KÖSTLIN, K. Die Regionalisierung von Kultur. In: KÖSTLIN, K. \& BAUSINGER, H. (Hg.). Heimat und Identität. Probleme regionaler Kultur. Neumünster: Verhandlungen des 22. Deutschen Volkskunde-Kongresses, 1980, p. 25-38. 
KÖSTLIN, K. Horti Oia und Mittelkärntner Blondvieh: Vom Genuss des Verräumlichten. Raum, 2010, Nº 80, p. 20-24.

LAHNER, M. Regional Governance in Biosphärenreservaten. Eine Analyse am Beispiel der Regionen Rhön und Schalsee unter Einbeziehung von Place-Making. Stuttgart: ibidem-Verlag, 2009.

MANSCHWETUS, U. Regionalmarketing. Wiesbaden, 1995.

MORE-HOLLERWEGER, E. \& HEIMGARTNER, A. Freiwilliges Engagement in Österreich. Studie im Auftrag des Bundesministeriums für Arbeit, Soziales und Konsumentenschutz. Wien, 2009.

MOSER, R. Der Biosphärenpark Großes Walsertal. Aktuelle Problemstellungen und Perspektiven aus Sicht der Praxis. In: COY, M. \& WEIXLBAUMER, N. (Hg.). Der Biosphärenpark als regionales Leitinstrument: Das Große Walsertal im Spiegel seiner Nutzer. Alpine space - man \& environment, 2009, Vol. 10, p. 109-122.

OEDL-WIESER, TH. Frauen und Politik: Ermächtigung und Partizipation. Raum, 2010, $N^{\circ} 77$, p. 30-32.

OSTROM, E. \& AHN, T.K. The meaning of social capital and its links to collective action. In: SVENDSEN, G.T. \& SVENDSEN, G.L.H. (editors). Handbook of Social Capital: The Troika of Political Science, Sociology and Economics. Cheltenham: Edward Elgar Publishing, 2009, p. 36-55.

PLASSER, F. \& ULRAM, P.A. Bürger und Politik in Österreich. In: GABRIEL, O.W. \& PLASSER, F. (Hg.). Deutschland, Österreich und die Schweiz im neuen Europa. Bürger und Politik. Baden-Baden: Nomos, 2010, p. 147-216.

PUTNAM, R.D. (Hg.). Gesellschaft und Gemeinsinn: Sozialkapital im internationalen Vergleich. Gütersloh: Verlag BertelsmannnStiftung, 2001.

PUTNAM, R.D. Bowling alone - The collapse and revival of American Community. New York: Simon \& Schuster, 2000.
RENN, O. \& KASTENHOLZ, H.G. Ein regionales Konzept nachhaltiger Entwicklung. GAIA, 1996, Vol. 5, № 2, p. 86-102.

REUTZ-HORNSTEINER, B. Der Biosphärenpark Großes Walsertal. Die Ausgangslage - Entstehung und Umsetzung. In: In: COY, M. \& WEIXLBAUMER, N. (Hg.). Der Biosphärenpark als regionales Leitinstrument: Das Große Walsertal im Spiegel seiner Nutzer. Alpine space - man \& environment, 2009, Vol. 10, p. 17-30.

REUTZ-HORNSTEINER, B. Sechs Gemeinden und ein Zukunftsprinzip - die Modellregion Biosphärenpark Großes Walsertal. SIR-Mitteilungen und Berichte, 2006, No 32, p. 67-74.

RUMPOLT, P.A. Das Selbstbild im Biosphärenpark Großes Walsertal. In: COY, M. \& WEIXLBAUMER, N. (Hg.). Der Biosphärenpark als regionales Leitinstrument: Das Große Walsertal im Spiegel seiner Nutzer. Alpine space - man \& environment, 2009, Vol. 10 , p. $43-62$.

SCHERMER, M. Re-lokalisierte Lebensmittelerzeugung: Oft mehr Wertschätzung als Wertschöpfung. Raum, 2010, Nº 80, p. 25-27.

STOLL-KLEEMANN, S. \& WELP, M. Participatory and Integrated Management of Biosphere Reserves. GAIA, 2008, Vol. 17, № 51, p. 161-168.

TOBEN, CH. Integrierte Regionalentwicklung und Regionalmanagement. Bonn, 2007.

TRATTNIGG, R. Sustainable Development and Public Participation. In: BRIX, E.; NAUTZ, J. \& WUTSCHER, W. (editors). State and Civil Society. Vienna: Passagen Verlag, 2008a, p. 197-220.

TRATTNIGG, R. Zukunftsfaktor Sozialkapital - Praktische Überlegungen zu Staat und Zivilgesellschaft in Österreich. In: BUßJÄGER, P. (Hg.). Sozialkapital, regionale Identität und Föderalismus. Wien: Braumüller, 2008b, p. 77-91. 
WEIXLBAUMER, N. Walserstolz: Ein Bergkäse beweist Regional Governance. Raum, 2010, No 80, p. 37-39.

WEIXLBAUMER, N. \& COY, M. Selbstund Fremdbild in der Gebietsschutzpolitik. Das Beispiel des Biosphärenparks Großes Walsertal. In: MOSE, I. (Hg.). Wahrnehmung und Akzeptanz von Großschutzgebieten. Oldenburg: BIS-Verlag, 2009, p. 37-57. 